\title{
Experimental Verification of Reflectionless Wide-Angle Refraction via a Bianisotropic Huygens' Metasurface
}

\author{
Michael Chen*11, Elena Abdo-Sánchez112, Ariel Epstein 3, and George V. Eleftheriades 1] \\ (1) The Edward S. Rogers Sr. Department of Electrical and Computer Engineering, University of Toronto, \\ Toronto, ON, Canada M5S 2E4 \\ (2) Dpto. Ingeniería de Comunicaciones, E.T.S.I. Telecomunicación, Universidad de Málaga, \\ Andalucía Tech, E-29071 Málaga, Spain \\ (3) Andrew and Erna Viterbi Faculty of Electrical Engineering, Technion - Israel Institute of Technology, Haifa 3200003, Israel
}

\begin{abstract}
We report the design, fabrication, and characterization of bianisotropic Huygens' metasurfaces (BHMSs) for refraction of normally incident beams towards 71.8 degrees. As previously shown, all three BHMS degrees of freedom, namely, electric polarizability, magnetic polarizability and omega-type magnetoelectric coupling, are required to ensure no reflections occur for such wide-angle impedance mismatch. The unit cells are composed of three metallic layers, yielding a printed-circuit-board (PCB) structure. The fabricated BHMS is characterized in a quasi-optical setup, used to accurately assess specular reflections. Subsequently, the horn-illuminated BHMS' radiation pattern is measured in a far-field chamber, to evaluate the device's refraction characteristics. The measured results verify that the BHMS has negligible reflections, and the majority of the scattered power is coupled to the desirable Floquet-Bloch mode. To the best of our knowledge, this is the first experimental demonstration of such a reflectionless wide-angle refracting metasurface.
\end{abstract}

\section{Introduction}

Engineered plane-wave refraction was one of the first functionalities demonstrated with a metasurface [1]. These ultrathin planar devices are comprised of subwavelength polarizable particles (meta-atoms), allowing interaction with applied fields on a subwavelength scale via equivalent boundary conditions [2,3]. It was soon found that to efficiently couple the incident beam towards a given direction in transmission, meta-atoms with both electric and magnetic polarizabilities have to be used [4-6]. Nonetheless, these so-called Huygens' metasurfaces (HMSs) feature a symmetric structure [5], which only allows matching the wave impedance of either the incident or refracted waves [7]. Due to the inevitable mismatch, specular reflections occur, becoming significant for wide-angle refraction [6].

This issue was solved in [8]; by breaking the symmetry of HMS meta-atoms, a metasurface that is impedance matched for both incident and refracted fields was devised. This con-

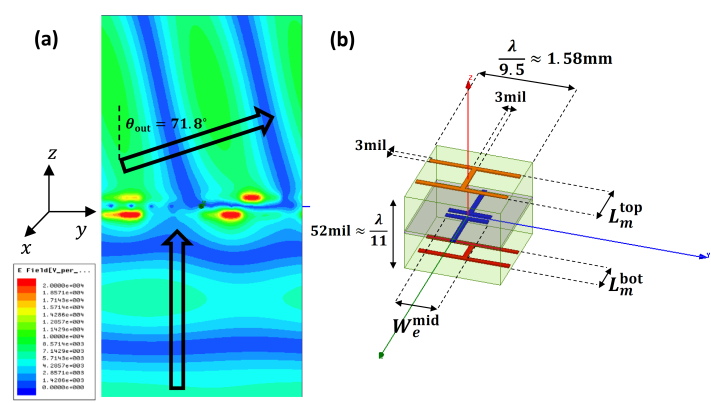

Figure 1. (a) Refracting BHMS (one period) and simulated field distribution $\Re\left\{\left|E_{x}(y, z)\right|\right\}$ [a.u.]. (b) Meta-atom geometry and parameters.

cept was later generalized in [9], showing that the required asymmetric meta-atoms feature omega-type bianisotropy (this was independently derived in [10]), exhibiting magnetoelectric coupling in addition to electric and magnetic polarizability. Correspondingly, it was shown that these bianisotropic Huygens' metasurfaces (BHMSs) can be realized by asymmetric cascade of three impedance sheets [8,9].

In this paper, we verify this theoretical concept, by designing, fabricating, and characterizing a BHMS for reflectionless refraction of a transverse-electric normally-incident beam $\left(\theta_{\text {in }}=0\right)$ towards $\theta_{\text {out }}=71.8^{\circ}$ at $f \sim 20 \mathrm{GHz}$ [Fig. [1.a)]. The design followed [9], yielding a printed circuit board (PCB) layout for the BHMS, verified via full-wave simulations. The fabricated PCB was then characterized in a quasi-optical setup, accurately assessing specular reflections. To complement this, the horn-illuminated BHMS' radiation pattern was measured in a far-field chamber, evaluating its refraction properties. The measured results indicate that, indeed, the specular reflections from the BHMS are negligible, and that the majority of the scattered power ( $\sim 80 \%)$ is coupled to the desirable Floquet-Bloch (FB) mode, propagating towards $71.8^{\circ}$. To the best of our knowledge, this is the first experimental demonstration of such a reflectionless wide-angle refracting metasurface; it verifies the theory as well as demonstrates the viability of PCB BHMSs for realizing future omega-bianisotropic devices [9-12]. 


\section{Theory, Design, and Physical Realization}

The derivation in [9] formulates the spatially-dependent electric, magnetic, and magnetoelectric responses required for implementing the desirable functionality. In the specific case of plane-wave refraction, this response can be naturally expressed via generalized scattering matrix $[\mathbf{G}]$ parameters, with the port impedances corresponding to the wave impedances of the incident and refracted modes [8,9]. Within this framework, the BHMS should be composed of meta-atoms at positions $y$, which exhibit unity (generalized) transmission coefficients $\left|G_{21}\right|=1$ and linear (generalized) transmission phase $\angle G_{21}(y)=-\frac{2 \pi}{\lambda} y \Delta_{\text {sin }}+\xi_{\text {out }}$, where $\lambda$ is the wavelength, $\xi_{\text {out }}$ is a constant phase, and $\Delta_{\text {sin }}=\sin \theta_{\text {out }}-\sin \theta_{\text {in }}$. This guarantees perfect impedance matching at both ports, while providing the necessary change in the transverse wavenumber to fully-couple the incident wave to the refracted one.

To implement the BHMS, the cascaded impedance sheet scheme of [8, 9] was used. Specifically, the BHMS consists of three copper layers $(1 / 2 \mathrm{oz}$.$) , defined on$ two 25mil Rogers RT/duroid 6010 laminates; the latter are bonded using a 2mil Rogers 2929 bondply, yielding an overall metasurface thickness of $52 \mathrm{mil} \approx \lambda / 11$ at the design frequency $f=20 \mathrm{GHz}$. Every meta-atom has $\Delta_{x} \times \Delta_{y}=\lambda / 9.5 \times \lambda / 9.5 \approx 1.58 \mathrm{~mm} \times 1.58 \mathrm{~mm}$ lateral dimensions, such that the metasurface period $\lambda / \Delta_{\text {sin }}$ contains 10 unit cells. Each unit cell consists of a dogbone, a loaded dipole, and another dogbone, forming the bottom, middle, and top impedance sheets, respectively [Fig. 11(b)]. The meta-atom response is controlled by the dogbone arm lengths, $L_{m}^{\text {bot }}$ and $L_{m}^{\text {top }}$, and the capacitor width $W_{e}^{\text {mid }}$. These parameters were initially set for each meta-atom following analytical formulas relating the required BHMS parameters to the sheet impedances, and fine tuned via simulations (ANSYS HFSS) to achieve the required overall bianisotropic response [9].

The final element geometrical parameters are presented in Fig. 2(a), along with the desirable and achievable responses [Fig. 2(b)-(c)], which generally agree well (losses cause reduction in $\left.\left|G_{21}\right|\right)$. Due to losses exhibited by the metaatoms with $\angle G_{21}$ around $0^{\circ}$, we used at two occasions identical unit cells (cells $\{\# 1, \# 2\}$ and $\{\# 3, \# 4\})$ that implement the average response of two consecutive elements, which improved the overall transmission.

One period of the metasurface was simulated under periodic boundary conditions, yielding the field distribution presented in Fig. 11a). Simulations predict that about $28 \%$ of the incident power is absorbed in the metasurface. Out of the scattered power, $93 \%$ is coupled to the desirable FB mode (transmitted towards $71.8^{\circ}$ ), $3 \%$ is specularly reflected, and $4 \%$ is transmitted towards $-71.8^{\circ}$. A frequency scan reveals that specular reflections are minimal at $19.8 \mathrm{GHz}$; however, the main device characteristics, i.e. the refraction efficiency and ohmic losses, are similar

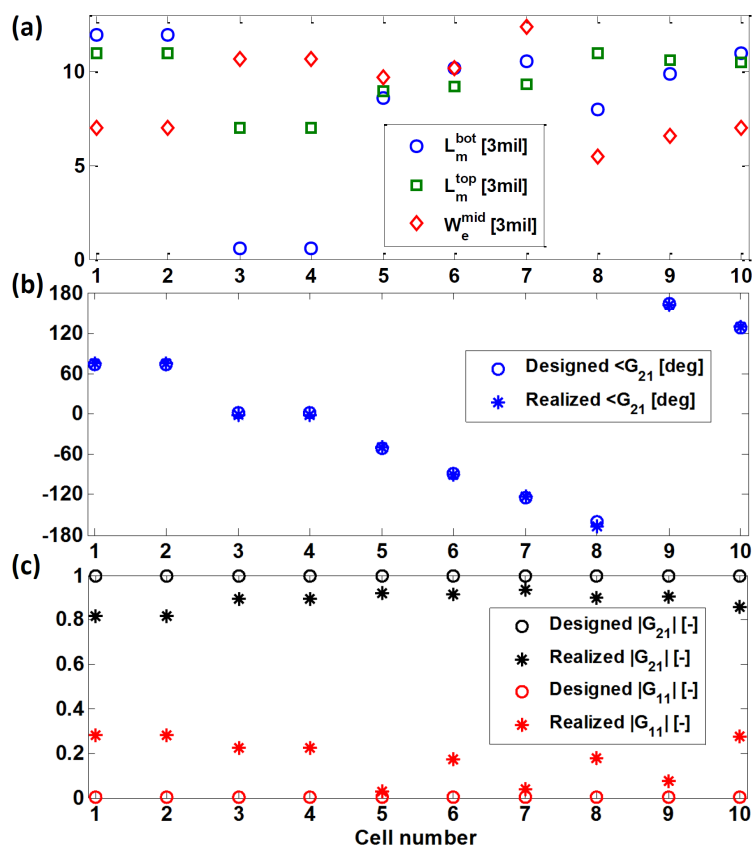

Figure 2. BHMS design. (a) Optimized geometrical parameters of the meta-atoms [corresponding to Fig. 11(b)]. (b) Designed (o) and realized $(*)$ generalized transmission phase. (c) Designed (o) and realized $(*)$ generalized transmission (black) and reflection (red) magnitudes.

within the range $19.8-20.0 \mathrm{GHz}$. This verifies that the designed BHMS indeed implements reflectionless wide-angle refraction as prescribed. The relatively high losses probably originate in the resonant nature of the meta-atoms, and could be improved by additional impedance sheets [13].

Following the verified design, a metasurface $\mathrm{PCB}$ of size $12^{\prime \prime} \times 18^{\prime \prime}=30.48 \mathrm{~cm} \times 45.72 \mathrm{~cm} \approx 20 \lambda \times 30 \lambda$ was fabricated (Fig. 3), containing $29 \times 190$ replicas of the simulated BHMS period [Fig. 11a)] along the $y$ and $x$ axes, respectively.

\section{Experimental Results}

\subsection{Quasi-optical Specular Reflection Exper- iment}

To test the specular reflectionless nature of the metasurface, a quasi-optical experiment was designed. This setup uses a horn (A-info LB-OMT-150220) and a Rexolite lens to focus a Gaussian beam onto the BHMS (Fig. 3). The horn and the BHMS are placed at the focal planes of the lens; thus, the wavefront incident upon the BHMS is planar, allowing the characterization to closely resemble theory and simulations.

The measured and simulated $\left|S_{11}\right|$ versus frequency are presented in Fig. 4, revealing a shift of the resonant frequency from $19.8 \mathrm{GHz}$ (simulated) to $20.6 \mathrm{GHz}$ (measured), which is attributed to fabrication errors and material uncertainties. The measured $S_{11}$ indicates that less than $0.2 \%$ of the in- 


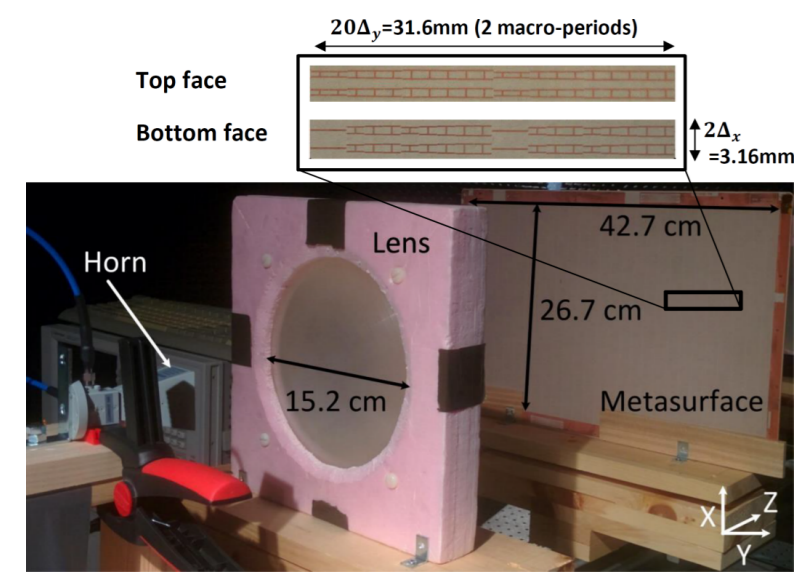

Figure 3. Quasi-optical experimental setup. The focal distances from the lens to the horn and from the lens to the BHMS are $12.5 \mathrm{~cm}$ and $29 \mathrm{~cm}$, respectively. Inset: Closeup on the top and bottom faces of the fabricated metasurface.

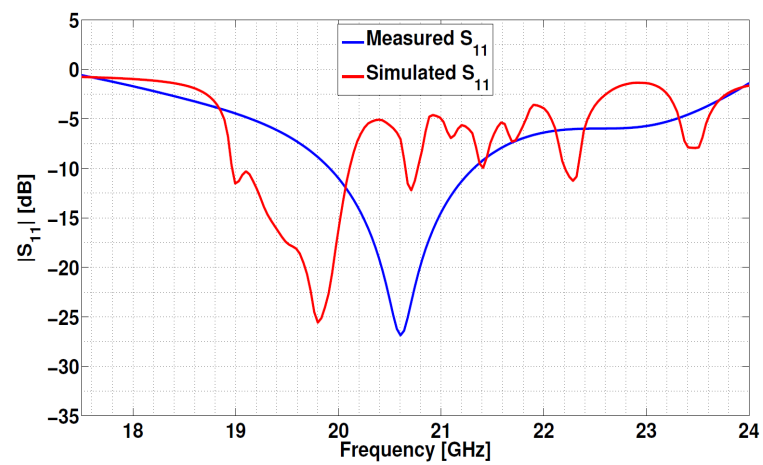

Figure 4. Measured and simulated normally incident specular reflection.

cident power is back-reflected, in agreement with simulations, verifying that the BHMS is indeed specularly reflectionless.

\subsection{Far-field Anechoic Chamber Refraction Experiment}

As the main feature of the metasurface is its ability for extreme refraction, an experiment was conducted to characterize this effect. To this end, the BHMS was placed in front of a standard gain horn antenna (Quinstar QWHKPRS-00), and the radiation pattern of the overall system (horn+BHMS) was measured as a combined antenna under test (AUT). The transmitting horn was placed sufficiently far from the BHMS to produce a planar wavefront on its $z \rightarrow 0^{+}$face, while the receiving horn was aligned behind the BHMS, facing its $z \rightarrow 0^{-}$face (see coordinate system in Fig. 1). Absorbers were attached to the back and sides of the receiving horn to reduce spurious scattering. The AUT was then rotated around its axis, and the gain pattern was measured, expected to yield a maximum around $\theta_{\text {out }}=71.8^{\circ}$.

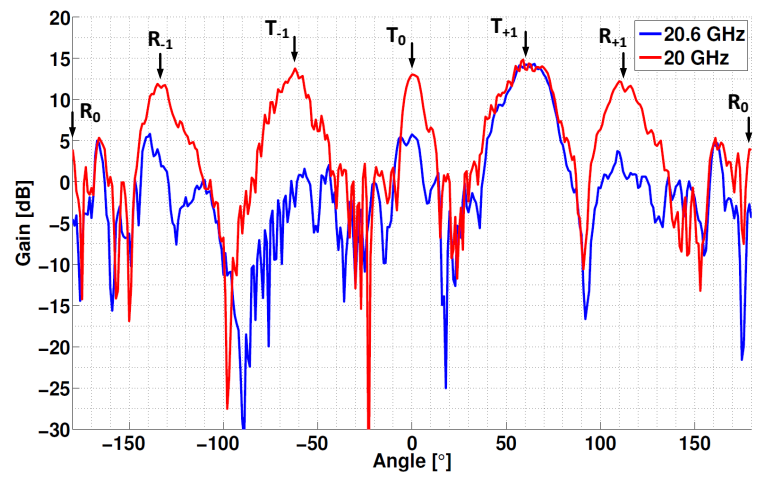

Figure 5. Measured AUT radiation patterns at $20.6 \mathrm{GHz}$ (resonant) and $20 \mathrm{GHz}$ (off resonance). The excited FB modes are clearly identified: the 0 th transmitted mode $\left(0^{\circ}\right)$, the \pm 1 transmitted modes $\left( \pm 71.8^{\circ}\right)$, the 0 th reflected mode $\left( \pm 180^{\circ}\right)$ and the \pm 1 reflected modes $\left( \pm 108.2^{\circ}\right)$.

We note that this test is not ideal and there are clear tradeoffs in the experimental setup. One such parameter is the distance of the receiving horn from the BHMS. As the BHMS was designed to interact with plane waves, the horn should be placed sufficiently far away to match the expected planar wavefront of the refracted wave. However, since the BHMS has a finite size, if the horn is placed too far, it would not be sufficiently shadowed by the surface. A good compromise was found at a horn-BHMS separation distance of $24 \mathrm{~cm}=16 \lambda$.

The measured radiation patterns at $20 \mathrm{GHz}$ and $20.6 \mathrm{GHz}$ are presented in Fig. 5. While the entire measured angular range is shown, the most reliable information is obtained between $-150^{\circ}$ and $150^{\circ}$. When the AUT is measured close to $\pm 180^{\circ}$, the receiving horn is partially blocking the line of sight between the transmitter and the metasurface; as the back of the horn has been fitted with absorbers, this would interfere with the measured results. Nonetheless, the quasioptical measurements complement the radiation pattern for these angles, assessing the specular reflection (Section 3.1.).

The radiation patterns in Fig. 5 clearly indicate that the BHMS implements the desirable refraction functionality. At $20 \mathrm{GHz}$, away from the (measured) resonant frequency, all propagating FB modes are excited, corresponding to multiple scattered beams. However, at resonance $(20.6 \mathrm{GHz})$, most of the scattered power is coupled to the designated mode, refracting towards $\approx 71.8^{\circ}$, while the other modes are suppressed.

A closer look at the pattern at $20.6 \mathrm{GHz}$ reveals that the gain actually peaks at $62^{\circ}$. However, as the gain of finite apertures deteriorates with a $\cos (\theta)$ factor, we must account for it to properly assess the direction of the main beam. This evaluates the refraction angle at $69^{\circ}$, much closer to the designated one; the deviation may originate in fabrication tolerances and alignment errors. The $3 \mathrm{~dB}$ beamwidth is $21^{\circ}$, corresponding to an effective aperture length of $20 \mathrm{~cm}$. 
Given the receiving horn dimensions and position, the expected $-10 \mathrm{~dB}$ beam diameter on the BHMS is $\approx 23 \mathrm{~cm}$ [14], in a good agreement.

Comparing the power in the refracted beam, calculated by integrating the gain between its nulls, to the overall integrated gain, indicates that approximately $80 \%$ of the scattered power is coupled to the desirable FB mode. Although this is smaller than the simulated $93 \%$, which may be attributed to fabrication and material tolerances, it still demonstrates a reasonable quantitative agreement with the designated device functionality. It should be noted that these efficiencies, corresponding to realistic BHMSs, are considerably higher than the theoretical $73 \%$ predicted for an ideal non-bianisotropic HMS implementing the same wide-angle refraction [6, 7]; this highlights the crucial role omega-type bianisotropy plays in face of a significant impedance mismatch.

Figure 5 further indicates that the suppressed scattering at $20.6 \mathrm{GHz}$ is mostly an outcome of increased absorption (the peak gain remains similar). Although this implies that losses become much pronounced at the resonant frequency, the current measurement setup does not allow reliable quantification of this parameter; this is left for future work.

\section{Conclusion}

We have demonstrated the first PCB BHMS implementing reflectionless wide-angle refraction. Experimental validation was carried out by combining the results of a quasioptical setup and radiation pattern measurements. The hybrid approach verifies that, indeed, specular reflections are negligible, and approximately $80 \%$ of the scattered power is coupled to the designated beam, albeit with considerable losses. Future work will include a more thorough quantification of losses, and using improved BHMS designs to further enhance the overall efficiency.

\section{References}

[1] N. Yu, P. Genevet, M. A. Kats, F. Aieta, J.-P. Tetienne, F. Capasso, and Z. Gaburro, "Light propagation with phase discontinuities: generalized laws of reflection and refraction," Science, 334, 6054, October 2011, pp. 333-7, doi:10.1126/science.1210713.

[2] C. L. Holloway, E. F. Kuester, J. A. Gordon, J. O'Hara, J. Booth, and D. R. Smith, “An overview of the theory and applications of metasurfaces: the twodimensional equivalents of metamaterials," IEEE Antennas and Propagation Magazine, 54, 2, April 2012, pp. 10-35, doi:10.1109/MAP.2012.6230714.

[3] S. A. Tretyakov, "Metasurfaces for general transformations of electromagnetic fields," Philosophical Transactions of the Royal Society of London A: Mathematical, Physical and Engineering Sciences, 373, 2049, July 2015, doi:10.1098/rsta.2014.0362.
[4] C. Pfeiffer and A. Grbic, "Metamaterial Huygens' surfaces: tailoring wave fronts with reflectionless sheets," Physical Review Letters, 110, 19, May 2013, p. 197401, doi:10.1103/PhysRevLett.110.197401.

[5] F. Monticone, N. M. Estakhri, and A. Alù, "Full control of nanoscale optical transmission with a composite metascreen," Physical Review Letters, 110, 20, May 2013, p. 203903, doi:10.1103/PhysRevLett.110. 203903.

[6] M. Selvanayagam and G. V. Eleftheriades, "Discontinuous electromagnetic fields using orthogonal electric and magnetic currents for wavefront manipulation," Optics Express, 21, 12, June 2013, pp. 1440914429, doi:10.1364/OE.21.014409.

[7] A. Epstein and G. V. Eleftheriades, "Floquet-Bloch analysis of refracting Huygens metasurfaces," Physical Review B, 90, December 2014, p. 235127, doi: 10.1103/PhysRevB.90.235127.

[8] J. P. S. Wong, A. Epstein, and G. V. Eleftheriades, "Reflectionless wide-angle refracting metasurfaces," IEEE Antennas and Wireless Propagation Letters, 15, April 2016, pp. 1293-1296, doi:10.1109/LAWP.2015. 2505629.

[9] A. Epstein and G. V. Eleftheriades, "Arbitrary powerconserving field transformations with passive lossless omega-type bianisotropic metasurfaces," IEEE Transactions on Antennas and Propagation, 64, 9, September 2016, pp. 3880-3895, doi:10.1109/TAP. 2016.2588495 .

[10] V. S. Asadchy, M. Albooyeh, S. N. Tcvetkova, A. Díaz-Rubio, Y. Ra'di, and S. A. Tretyakov, "Perfect control of reflection and refraction using spatially dispersive metasurfaces," Physical Review B, 94, August 2016, p. 075142, doi:10.1103/PhysRevB.94.075142.

[11] V. S. Asadchy, Y. Ra'di, J. Vehmas, and S. A. Tretyakov, "Functional metamirrors using bianisotropic elements," Physical Review Letters, 114, March 2015, p. 095503, doi:10.1103/PhysRevLett. 114.095503 .

[12] A. Epstein and G. V. Eleftheriades, "Synthesis of passive lossless metasurfaces using auxiliary fields for reflectionless beam splitting and perfect reflection," Physical Review Letters, 117, December 2016, p. 256103, doi:10.1103/PhysRevLett.117.256103.

[13] C. Pfeiffer and A. Grbic, "Bianisotropic metasurfaces for optimal polarization control: Analysis and synthesis," Physical Review Applied, 2, October 2014, p. 044011, doi:10.1103/PhysRevApplied.2.044011.

[14] P. F. Goldsmith, Quasioptical systems: Gaussian beam quasioptical propagation and applications, chapter 7, IEEE press, 1998, pp. 178-179. 